\title{
The Rising Prospects of Cloud Robotic Applications
}

\author{
S. Jordán*, T. Haidegger**, L. Kovács**, I. Felde** and I. Rudas** \\ * Budapest University of Technology and Economics (BME) \\ ** Óbuda University (ÓU) \\ jorsa89@gmail.com, \{haidegger,rudas\}@uni-obuda.hu, \{kovacs.levente, felde.imre\}@nik.uni-obuda.hu
}

\begin{abstract}
Cloud Robotics is an emerging field within robotics, currently covering various application domains and robot network paradigms. This paper provides a structured, systematic overview of the numerous definitions, concepts and technologies linked to Cloud Robotics and cloud technologies in a broader sense. It also presents a roadmap for the near future, describing development trends and emerging application areas. Cloud Robotics may have a significant role in the future as an explicitly human-centered technology, capable of addressing the dire needs of our society.
\end{abstract}

Keywords—cloud robotics, distributed systems, cloud computing, networked robotics

\section{INTRODUCTION}

Cloud Robotics (CR) was born from the merger of cloud technologies and service robotics, which was preceded by a change in paradigm in both domains. Cloud technology-based computing-or simply Cloud Computing - is one of the most dynamically growing areas of Info-Communication Technologies (ICT). Cloudbased solutions gradually appear in ICT areas with wide user pool, such as Google Docs [1], which provides access to cloud-based software and storage, as an example of Software as a Service (SaaS) [2]. Similarly, entire portfolio of services is provided as Platform as a Service (PaaS) or Infrastructure as a Service (IaaS) to companies and larger institutions. Based on these, a new term appeared, Robots as a Service (Raas), where robotic systems are involved. It is common in all these models that they aim to dynamically link the service to the user at various complexity levels. On the top of the hierarchy sits Cloud Robotics, a new paradigm and a System of Systems in itself [3].

The relationship between the models above is shown in Fig. 1. The American National Institute of Standards and Technology (NIST) has defined firmly the concept of cloud technology based on these models: "Cloud computing is a model for enabling ubiquitous, convenient, on-demand network access to a shared pool of configurable computing resources (e.g., networks, servers, storage, applications and services) that can be rapidly provided and released with minimal management effort or service provider interaction"[4]. A cloud (sub-cloud) can be private, public, community or hybrid-which is a composition of two or more cloud-deployments type. The five essential characteristics representing the basis of understanding of CR are the following:

- On-demand self-service,

- Broad network access,
- Resource pooling,

- Rapid elasticity,

- $\quad$ Measured service.

Along ICT, robotics by itself has also developed a lot, and unquestionably service robotics is becoming the leading area within. The shift of paradigm from rigid industrial manipulators as the etalon of robots to more versatile, human-centered devices is clearly traceable on the standardization efforts. While the original International Standardization Organization (ISO) $8373: 1994^{1}$ only dealt with industrial manipulators, not allowing any humans to be in the vicinity of the operating robot (must had been protected by fences, rails, light curtains, etc.). The new, 2007 edition $^{2}$ already allows the mixed environment of robots, and the first standard addressing the safety issues of human-robot collaboration (ISO 13482:2013 ${ }^{3}$ ) will appear later this year. The definition of robot has also changed in the meanwhile, allowing a wide range of service robots to be called robot, or robotic device: "a robot that performs useful tasks for humans or equipment excluding industrial automation applications, while personal service robot used for a noncommercial task, usually have lower requirements ${ }^{2 \prime}$. This evolution opened the field for new service-based models.

\section{THE BIRTH OF CLOUD ROBOTICS}

The term "cloud-enabled robotics" was presented by James Kuffner for the first time at the IEEE RAS Intl. Conference on Humanoid Robotics in 2010 [5]. He was first to point out the potential of distributed networks combined with robotics, primarily to enhance the robot

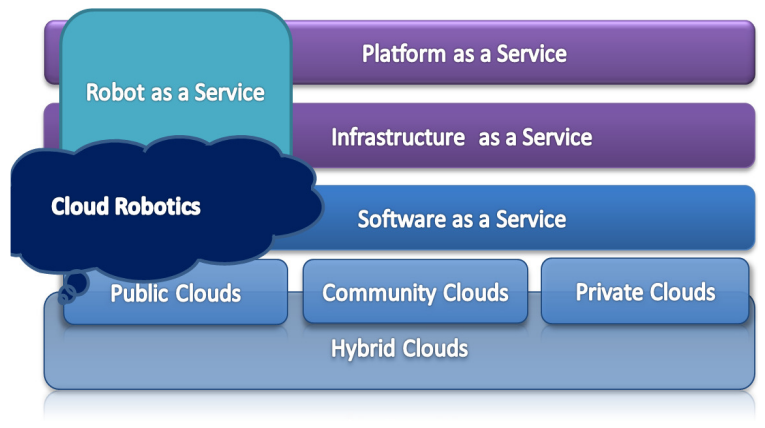

Figure 1. Cloud computing service models, extended with the service layers provided on the top of the cloud, topped by the concept of Robot as a Service and Cloud Robotics.

\footnotetext{
${ }^{1}$ ISO 8373:1994, Manipulating industrial robots-Vocabulary ${ }^{2}$ ISO 8373:2012, Robots and robotic devices-Vocabulary

${ }^{3}$ ISO/FDIS 13482 Robots and robotic devices-Safety requirements for non-industrial robots-Non-medical personal care robot
} 

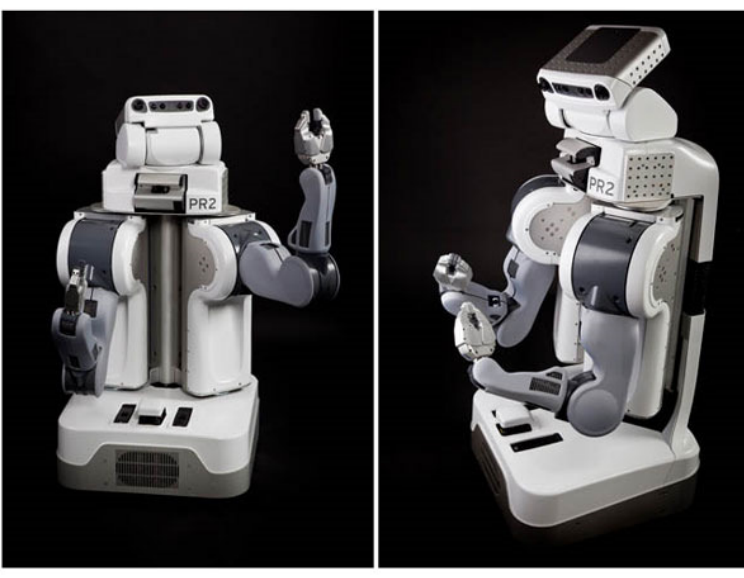

Figure 2. PR2 is a generic research platform widely used for cloud applications. The PR2 has demonstrated various capabilities relying on the Robot Operating System (ROS) [6].

agents limited capabilities. CR presented a need of fast, reliable and ubiquitous network connections. The internet infrastructure has gone through a radical change in the past 10 years in terms of bandwidth and Quality of Service, and is now able to enable to support the cloud reliably. In 2011, at the Google I/O developer Conference, Google and Willow Garage introduced their theory and foreseen application of CR [7]. Using the PR2 research robot, it was first demonstrated how to make robots smarter and more energy-efficient with these techniques (Fig. 2).

Cloud robotics can become the "remote-brain" of the agents, freeing the hardware from computationalexpensive tasks. However, this is not an entirely new concept: back in 1990, Inaba already presented the concept of "Remote Brained Approach", as he imagined a server farm or even a human operator who remotely gives instructions to a robot, i.e., an agent [8].

\section{DEFINING CLOUD ROBOTICS}

There are several definitions of CR floating around, and various labs use them in quite different senses. Ken Goldberg (UC Berkeley) emphasizes the benefits of the unlimited computation capacity, memory and programming capability, leading to a new form of collective robot intelligence through learning and sharing $[9,10]:$ "Humans as a species are getting smarter because we are able to share information much quicker and build on innovations faster, robots have that potential as well" [11].

The same research group has described more specifically that "Cloud Robotics proposes a thin-client model where robots are connected to modern cloudcomputing infrastructure for access to distributed computing resources and datasets, and the ability to share training and labeling data for robot learning"[12].

According to Steve Cousins (CEO of Willow Garage Inc.) the role of cloud robotics is to provide an opportunity for open communication among robots, since "No robot is an island" [11]. The transfer medium is the wireless connection, just like the air for human communication, and robots use bits and bytes instead of words and sentences.

Other groups are convinced that $\mathrm{CR}$ can expand the robots' knowledge and skills and also make them more efficient. The RoboEarth EU FP7 project focuses on the research of these technologies, emphasizing the importance of cloud-based robotics [13]. They placed the extension of robot knowledge on two pillars: cloud computing and computer science, through which big data processing is supported. These ideas are implemented in their "World Wide Web for robots" concept, which is basically a network and database repository. Thus, robots can collect and share knowledge with each other through the network. One of the most profound definitions was given by Mario Tremblay: "Cloud robotics happens when we connect robots to the internet and then, by doing so, robots become augmented with more capacity and intelligence. The cloud lets them communicate with other machines and serve their human operators better. Connected robots equal augmented robots. By collaborating with other machines and humans, robots transcend their physical limitations and become more useful and capable, since they can delegate parts of their tasks to more suitable parties" [14].

These definitions demonstrate the diversity of Cloud Robotics approaches within the community. We believe that CR is more than a mere network of robots or a System of System employing robots, since the communication and knowledge acquisition and sharing through the cloud is an inherent design property of the system, and is exploited extensively to solve the desired tasks.

\section{A. Division of Cloud Technologies Regarding Robotics}

Cloud robotics can be divided into several areas, which are not easy to separate, since some are used as references to the entire sub-field, while others are building components only, yet all are using various features of the cloud, as presented below.

- One of the most basic type of cloud-based robotics focuses on the network such as an infrastructure. This is the Cloud Network (CN), which is basically divided into three subtypes:

○ In the case of Teleoperated Robots, the control is provided by a human operator who may be sitting next door, or on another continent. The goal is to achieve telepresence (fully capable virtual representation), as well as to perform complex tasks. The teleoperation has significant use in disaster relief, remote exploration and telerobotic mining. One of the major application areas is medicine. Besides successful telediagnostic services, the da Vinci Surgical System [15] has become prevalent in minimally invasive urological and gyneacological procedures, and the concept of telemedicine is expandable event to extra-planetary health care [16]. The main challenges in teleoperated robotics are the network reliability and communication delay, since the quality of the human-machine communication $(\mathrm{H} 2 \mathrm{M})$ determines the effectiveness of the task execution [17].

- Multi-robot systems are essentially based on the cooperation of robots without human intervention [18]. (E.g., a robot soccer team where individual 
agents work together in order to achieve a goal.) With this method, the resources of the robots can be better utilized. In this domain, the main challenge is the most efficient allocation of tasks among the participants. This typically requires effective resource allocation algorithms and machinemachine communication (M2M).

- Sensor arrays could be considered as a form of cloud systems (systems of systems), as long as robots are also involved in the architecture, where they are the recipients of information from the remote sensors via M2M communication. This method is useful for life support systems, in rescue robotic scenarios, or providing touring support service in shopping mall e.g. [19].

- Cloud Computing (CC) changes the fundamental components of today's ICT, snd whirling into the field of robotics as well. The CPU heavy tasks, such as image recognition, voice recognition, path planning can be performed in the cloud. This means that significantly cheaper hardware can be built into the agent, furthermore, the energy consumption can be reduced up to $40 \%$ [7]. The current main challenge of the field is that if the connection is interrupted with the cloud, the robot still needs to present basic functionality. Due to the fact that network transmission and data transfer can still take a lot of time, it does not prove practical to perform all computations in the cloud. There is a task and system dependent optimum regarding the robot's on-board computation and the machine-cloud communication (M2C) [18].

Cloud Storage (CS) is one of the key enabling components of cloud robotics. It is impossible to store all human knowledge in a single system, let alone, in a robot. An agent should be enabled through the cloud to acquire new knowledge. If the robot sees a new object, it may still be able to recognize it via semantic search through the cloud repository, which is identical and accessible to all robots. This is a next step towards cognitive robotics, and practically, it can save storage space and computing power, which leads to the decrease of costs. CS typically means M2C.

\section{SYSTEM EXAMPLES}

Today, one of the pioneering companies in the field is Willow Garage (Menlo Park, CA, USA), their multipurpose service robot is called PR2 [20]. The PR2 heavily relies on the Willow Garage-developed open-source software package, the Robot Operating System (ROS). It is a collection of libraries and tools what can make the work of developer easier and faster [21]. ROS can be considered to be a meta-operating system, and in the past years it has become by far the most wide-spread robot library.

A key enabling component of the ROS-based cloud is the rosbridge package that allows dynamic socket and web-socket based access to ROS, therefore realizing the communication between the robot and the ROS environment via the cloud [22]. Android-based systems enjoy great popularity these days, and the rosjava package can be useful as it connects Android and ROS-based robots [23]. The PR2 robot also builds on the Point Cloud
Library (PCL), another standalone, large scale, open project for effective 2D/3D image point cloud processing.

Google Inc. is big supporter of CR, aiming to become a leader in this domain. The rosjava package was developed at Google in cooperation with Willow Garage. In addition, Goggle's services-such as image recognition and navigation/mapping - are very useful for robotic applications. NASA is in collaboration with Google, working on Android-cloud-robot connection with Rover [24].

An education-oriented approach is demonstrated by the Arduino-based robots, compatible with ROS. Simple robots can easily be created, immediately make use of cloud services [25]. The computing power lays in the cloud so we can build cheap, yet capable robots similar to the "10 Dollar Robot Design Challenge" [26].

These concept allows for robots to share information and learn from another companion in a structured way, for which a high-end network infrastructure is essential. ASORO [27] and Gostai [28] developed cloud computing infrastructure which can serve as the basis of CR. Network capabilities have been tested through various events, notably, the Plugfest experiment in 2009, where telerobotic master and slave systems were connected in multiple setups for twenty-four hours [29]. The goal was to demonstrate the feasibility of internet-based telesurgery. DavinCi is a software framework that provides cloud-based services for robots [30]. Aforementioned RoboEarth is working on the World Wide Web for robots, building on big network theory and large database repositories.

The potential in the cloud has been recognized by large ICT companies as well, although, mostly addressing particular artificial intelligence (AI) problems, not fully belonging to robotics. For example, Apple's Siri voice recognition software uses the cloud to identify recorded sounds then contact the server for computational and database assistance.

In the meanwhile, Android-based devices are becoming more and more popular, and since they can be integrated into most applications in the cloud, it is expected that apps will be available very soon to empower and mechatronic structures to become a CR with the touch of a button. The smart phone can really act as the "brain" of the robot [31] (Fig. 3).

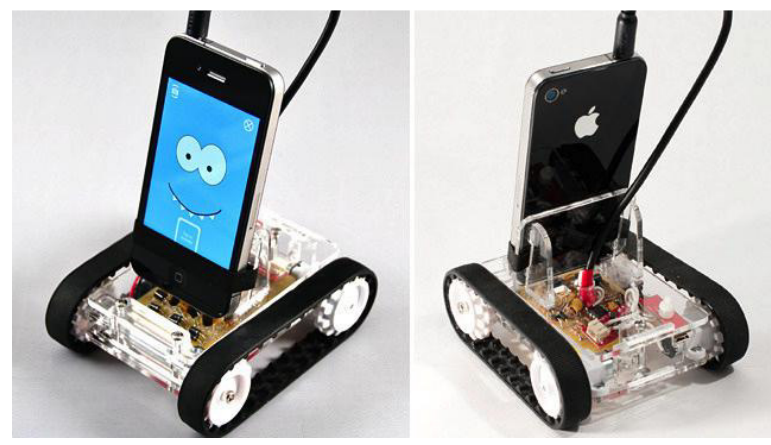

Figure 3. Romo is controlled and powered by an iOS device which can connect to the cloud. [32] 


\section{RISING APPLICATIONS OF CLOUD ROBOTICS}

Unquestionably, $\mathrm{CR}$ is a fascinating research topic, yet it has to identify its target applications and finds its particular domains. It is believed that $\mathrm{CR}$ is a way to introduce cognition into the domain of robotics, which is envisioned and strongly supported by the European Union through the FP calls.

In a human-centered, real world environment, the robot can encounter a lot of computation-intensive tasks. One of the most common problems is the recognition and transfer of an unknown object. This is a basic human action, yeteven nowadays-it is a complex and difficult task to be carried out by a home robot. Willow Garage's PR2 performed this task employing cloud-based technologies in the following sequence:

1. Capture data with robot: This takes place on board of the robot which detects the object with a 2D camera and 3D scanner. This produces a 2D image and a 3D point cloud, but only the $2 \mathrm{D}$ image is sent for processing to the cloud server.

2. The processing of data on cloud servers: If the server was able to identify an object based on the 2D image, it returns a 3D CAD model of the object to the robot. In the case of PR2, the Google Goggles online application can provide the image processing unit for this service [33]. It is also available on Android platform, therefore it can be used widely.

3. The robot applies the processed data: Based on the obtained 3D CAD model and the measured 3D point cloud, the robot determines the object's position and orientation. The robot applies the grasping movement. (In the demo, this action was carried out by Columbia University's GraspIt! system [34].

4. Feedback to the server: Finally, the robot sends the results of the grasping action, so that train the server's database for further uses.

The process has online and offline elements, and it is based on both models.

CR has the potential to be applied to various tasks in a human-centered environment based on [12] including:

- Indexing, annotating large sets of databases, images, object models, verifying those through experience

- Cloud-based motion planning and uncertainty modeling

- Sharing task execution experience with other agents

- Still allowing human interaction and remote-guidance if needed.

A possible area where CR may appear is of home robots, built on the thin-client model, where the devices use the cloud infrastructure for distributed computing and storage. Experiments designed on the Google Object Recognition Engine and the PR2 robot have been conducted [12].

Two of the most promising application areas of service robotics are healthcare and homecare. In recent years, various new systems have emerged, supporting minimally-invasive telesurgery or telepresence robots acting as a remote agent for a visiting doctor. However, due to the criticality of the tasks (in the case of any adverse events), medical applications are reluctant to trust task-computation on the cloud. Nevertheless, homecare is a much more promising application, where many of the daily tasks of the aging people should be taken over by robots [35].

In the case of autonomous Unmanned Arial Vehicles (UAV), the cloud is an important element allowing the reduction of on-board computational requirement and thus the amount of battery [36]. When a fleet of drones are used, typically a larger vessel (such as the Airborne Warning and Control System (AWACS) for the US Air Force) provides the high-quality, high bandwidth, low latency communication infrastructure.

Another possible-yet far-fetched-domain of CR is space research. Since we have already deployed thousands of satellites and deep space vessels with various computational capabilities, it would be beneficial to recollected these devices into a distributed space computingnetwork, that could support e.g., the rovers on the Mars in data processing and situation analysis, or other future exploration tasks involving robots [17].

The cloud is not just a new solution for old tasks but provides new opportunities for research, development and education. It has become possible to access robots with Android devices or via the internet, which is a crucial factor for those, who cannot afford expensive hardware. Such cloud-centered education has been implemented at the University of Colorado, allowing remotely located students to participate [37]. The emerging trend of Massive Open Online Courses (MOOCs) began with the opening of the AI class at Stanford to the public in 2011, which attracted 60,000 people [38].

\section{A ROADMAP FOR CLOUD ROBOTICS}

As of last year, CR was one of the research focuses in robotics and the interest continues to rise [39]. The 2013 Roadmap for U.S. Robotics seriously counts on CR for future applications [40]. They identified five areas where the field will advance significantly.

1. Robots in the real-world need a lot of information, about the world, because there are limited in capacity to explore the environment. The recognition of various objects, sounds, images, people can be done much more efficiently with the help of the cloud.

2. Navigation of robots can move forward much because Google Maps-like service are fast and reliable so that the robots could exceed the limit of its on-board capacity. Cloud services can revolutionize modeling and motion planning, by reducing costs and increasing the innovation component.

3. The communication between robots will also evolve, becoming a crucial component of a System of Robots. Agents could share their sensory information, calculation results, news or their experience (advancing to cognitive level), gained through their reasoning skills. This application is going to be similar to s "Facebook for robots" where the other robots can share the records, so it is always the best option (chosen on the community level) that may be implemented by the single agent.

4. We need to encourage people to generate open-source codes, share their plans, experience and results. According to the U.S. Robotics Roadmap, a large growth of these communities is expected over the next 5 years. 


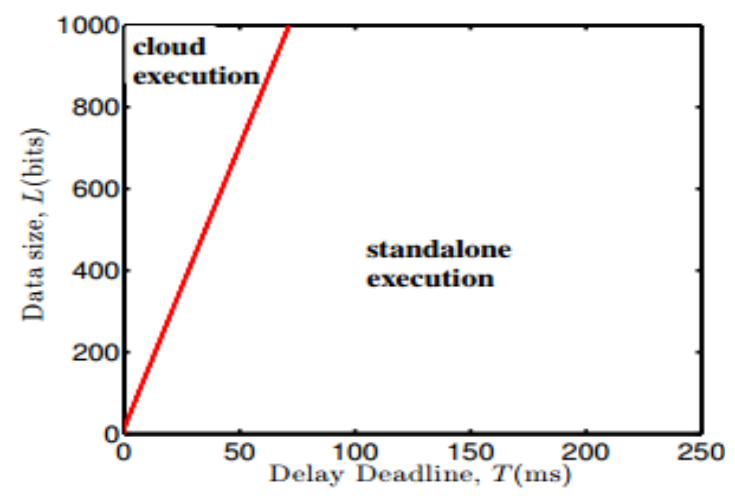

Figure 4. Tradeoff between computation and transmission time, a key design parameter for cloud robots [18].

5. The need of human work does not only manifest in the field of development because if we develop so autonomous robots then human operator may be necessary who co-ordinates the robot's behavior in the case of error.

Along these lines, it is expected that many applications will emerge from the users' side, in forms that are not necessarily belonging to robotics (as we see it today), yet bearing all the marks listed above.

\section{LIMITATIONS OF THE TECHNOLOGY}

One of the most important issues is about security resulting from the use of the wireless infrastructure. The robot sends and receives sensitive data which is essentially influencing its behavior. The security of the data on-board and transmitted information equally needs to be protected. This may contain private data, or critical information (e.g., regarding medical condition). Detailed information about people's behavior is already sensitive, and should not be exploited.

One of the essential questions of cloud robotics is what should be the optimal ratio between online and offline computation. If the robot relies too much on cloud services, it can become "brainless" when the network connection is lost for a longer time. Besides, transmitting large data over the network is taking time, just as onboard computation (Fig. 4.).

In addition, the network communication delay must be kept at a low level and we have to defend against possible packet loss with fault-tolerant protocols. The statistics collected by the Roadmap for U.S. Robotics shows that robotic manufacturing, service and medical applications have increased by an annual $20 \%$ on average, and will continue rising. These emerging domains will drive the development towards advanced supporting technologies.

\section{DISCUSSION}

Arguably, Cloud Robotics is an evolutionary jump for robots, a transformational change of paradigm, where the communication with peers (robot-to-humans) has become an essential characteristic of a system. It all has become possible through extension of physical limitation of a single agent, using the resources of the cloud.

At the current stage of development, robots can use their sensory information-including cameras, sonars, 3D scanners - to perform extended search on large data bases and the internet to identify any unknown object or situation they may encounter. Then, they use this online knowledge to create a plan how to deal with the situation. The results of the action are fed back to the cloud so other systems can immediately get access to that knowledge. This distributed problem-solving and knowledge-sharing is the essence of CR, and the approach could be extended generally to any CPU-heavy task.

In the foreseeable future, a swarm of simple robots and robotic devices will join a CR network, exploiting the advantage of cloud technologies. We shall witness the rise of distributed intelligence in robotics that allows the creation of a global database for navigation, mapping, planning task execution and beyond. The increasing number of robots with up to date knowledge will become a true helping hand for humans.

\section{ACKNOWLEDGMENT}

Levente Kovács was supported by the János Bolyai Research Scholarship of the Hungarian Academy of Sciences.

\section{REFERENCES}

[1] Cloud Tweaks, Cloud Computing and Google Docs. (2010, December 18) Available: www.cloudtweaks.com/2010/ 12/cloudcomputing-and-google-docs, 2010.

[2] I. Sriram and A. Khajeh-Hosseini, Research Agenda in Cloud Technologies. Methodology cs.DC: 1-11. Available: arxiv.org/abs/1001.3259, 2010.

[3] E. Guizzo, Robots with their heads in the clouds. IEEE Spectrum, vol. 48, issue: 3, pp. 16-48, 2011

[4] P. Mell and T. Grance, The NIST Definition of Cloud Computing. Recommendations of the National Institute of Standards and Technology. NIST Special Publication 145, vol. 6, no. 7, pp. 1-7, 2011. Available: http://csrc.nist.gov/publications/nistpubs/800145/SP800-145.pdf.

[5] J. Kuffner, Cloud-Enabled Robots. In proc. of the IEEE Intl. Conf on Humanoid Robots, Nashville, 2010.

[6] Robotic Evolution. ROS- the Artificial Intelligence for Robots Available: $\quad$ robotsfuture.blogspot.hu/2011/07/ros-artificialintelligence-for-robots.html, 2011.

[7] D. Kohler, R. Hickman, K. Conley and B. Gerkey, Cloud Robotics. Google I/O 2011 Developer Conference, 2011.

[8] M. Inaba, Remote-brained robots. In proc. of the Intl. Joint Conf. on Artificial Intelligence, vol. 15. Lawrence Erlbaum Associates Ltd, pp. 1539-1606, 1997.

[9] K. Goldberg. Cloud Robotics. Available: goldberg.berkeley.edu/ /cloud-robotics, 2013.

[10] K. Goldberg and B. Kehoe, Cloud Robotics and Automation: A Survey of Related Work. UC Berkeley Technical Report No. UCB/EECS-2013-5. Available: http://www.eecs.berkeley.edu/ /Pubs/TechRpts/ 2013/EECS-2013-5.html, 2013.

[11] K. Goldberg, Understanding no robot is an island. Humans Invent Available: goldberg.berkeley.edu/cloud-robotics, 2012.

[12] B. Kehoe, A. Matsukawa, S. Candido, J. Kuffner, K. Goldberg, Cloud-Based Robot Grasping with the Google Object Recognition Engine. IEEE International Conference on Robotics and Automation. (ICRA), Karlsruhe, pp. 4248-4256, 2013.

[13] RoboEarth, What is Cloud Robotics? Available: www.roboearth.org/cloud_robotics, 2013.

[14] RobotShop, Pioneers Cloud Robotics, Interview With Mario Tremblay. Available: www.robotshop.com/blog/en/myrobots-com -pioneers-cloud-robotics-interview-with-mario-tremblay-1348

[15] T. Haidegger, "The Advancement of Robotic Surgery—Successes, Failures, Challenges (in Hungarian)," Orvosi Hetilap, vol. 151, no. 41, pp. 1690-1696, 2010.

[16] T. Haidegger and Z. Benyó, Surgical robotic support for long duration space missions. Acta Astronautica. vol. 63, no. 7, pp. 996-1005, 2008. 
[17] T. Haidegger, L. Kovács, R.-E. Precup, B. Benyó, Z. Benyó and S. Preitl, "Simulation and control for telerobots in space medicine," Acta Astronautica, vol. 81, issue: 1, pp. 390-402, 2012.

[18] G. Hu, W. P. Tay and Yonggang Wen, Cloud Robotics: Architecture, Challenges and Applications. IEEE Network, no. 26, pp. 21-28, 2012.

[19] K. Kamei, S. Nishio, N. Hagita and M. Sato, Cloud Networked Robotics. IEEE Network, no. 6, pp. 28-34, 2012.

[20] Willow Garage, Overview Available: www.willowgarage.com/ /pages/pr2/overview, 2013.

[21] M. Quigley, B. Gerkey, K. Conley, J. Faust, T. Foote, J. Leibs, E. Berger, R. Wheeler and A. Ng, ROS: an open-source Robot Operating System. In proc. of the ICRA Workshop on Open Source Software, Kobe, vol. 32, pp. 151-170, 2009.

[22] ROS, Documentation Available: www.ros.org/wiki, 2013.

[23] ROS Java, Summary. Available: code.google.com/p/rosjava, 2013.

[24] RobotShop. Android + Arduino + ROS = Cloud Robotics Available: www.robotshop.com/blog/en/google-uses-android-tobring-arduino-into-the-clouds-1297, 2011.

[25] African Robotics Network, The AFRON "10 Dollar Robot" Design Challenge. Available: roboticsafrica.org/design_challenge.html, 2013.

[26] ASORO, Research. Available: www.asoro.astar.edu.sg/research_main.html, 2013.

[27] Gostai, GostaiNet. Available: www.gostai.com/ activities/consumer, 2013.

[28] H. H. King, B. Hannaford, K.-W. Kwok, G-Z Yang, P. Griffiths, A. Okamura, I. Farkhatdinov, et al., Plugfest 2009: Global interoperability in Telerobotics and telemedicine. In proc. of the IEEE Intl. Conf. on Robotics and Automation (ICRA), Anchorage, pp. 1733-1738, 2010.

[29] R. Arumugam, V. R. Enty, L. Bingbing et al. DAvinCi: A cloud computing framework for service robots. In proc. of the IEEE Intl.
Conf. on Robotics and Automation (ICRA), Anchorage, pp. 30843089, 2010.

[30] Übergizmo. Romo smartphone bot can double up as a spybot. Available: www.ubergizmo.com/2011/11/romo-smartphone-botcan-double-up-as-a-spybot, 2011.

[31] iDownloadBlog, Meet Romo, the iPhone-Powered Robot Available: www.idownloadblog.com/2011/11/27/romo/, 2011.

[32] SmartPlanet, Can cloud computing make robots smarter? Available: www.smartplanet.com/blog/smart-takes/can-cloudcomputing-make-robots-smarter/16382, 2011.

[33] Google, Google Goggles. www.google.com/mobile/goggles/\#text, 2013.

[34] Computer Science Department, Columbia University, GraspIt! Available: www.cs.columbia.edu/ cmatei/graspit, 2013.

[35] J. Cohn, The Robot Will See You Now. The Atlantic (March 2113). www.theatlantic.com/magazine/archive/2013/03/the-robotwill-see-you-now/309216, 2013.

[36] D. Stojcsics and A. Molnár, UAV Hardware and Software System for Small Size UAVs. Intl. J. of Advanced Robotic Systems, vol. 9, issue. 174, pp. 1-8, 2012.

[37] University of Colorado at Boulder, Real-Time Embedded Systems. Available: cuengineeringonline.colorado.edu/coursedb/ /view-course/435, 2013.

[38] J. Widom, From 100 student to 100,000. Available: wp.sigmod.org/?p=165, 2012.

[39] E. Guizzo. T. Deyle, Robotics Trends for 2012. IEEE Spectrum vol. 3, pp. 119-123, 2012.

[40] U.S. Robotics, A Roadmap for U.S. Robotics. Available: roboticsvo.us/sites/default/files/2013\%20Robotics\%20Roadmap-rs.pdf, 2013. 